\title{
Observation and reduction of mutual events in the solar system
}

\author{
B. Noyelles ${ }^{1}$, V. Lainey ${ }^{2,1}$ and A. Vienne ${ }^{1}$ \\ ${ }^{1}$ IMCCE - Laboratoire d'Astronomie de Lille, UMR8028 du CNRS, 1 impasse de \\ l'Observatoire, 59000 Lille, France email: Benoit.Noyelles@imcce.fr \\ ${ }^{2}$ Royal Observatory of Belgium, Ringlaan 3, B-1180 Brussels, Belgium
}

\begin{abstract}
Mutual event observations started in the early 1970s with the Galilean satellites. These observations were needed because of the Voyager spacecraft future arrival. Since 1979, IMCCE has organized observational campaigns for the Galilean satellites (called PHEMU), and since 1995 for the Saturnian satellites (also called PHESAT). Meanwhile, the reduction techniques have been greatly improved. Mutual event observations are one of the most accurate methods for obtaining positions of natural satellites, useful for detecting tidal effects. Hence mutual events of Jovian and Saturnian natural satellites are regularly observed around the world. This paper aims to describe mutual events and the advantages of this kind of observation besides the classical astrometric ones.
\end{abstract}

\section{What is a mutual event?}

There are two kinds of mutual events: occultations and eclipses. An occultation between two natural satellites occurs when the observer cannot distinguish the two satellites separately because a part of the further one is hidden by the nearest one (they are nearly aligned with the observer), whereas an eclipse occurs when the further satellite meets the umbra or the penumbra cone of the nearest one, so there is nearly an alignment between the Sun and the two satellites (see Fig. 1).

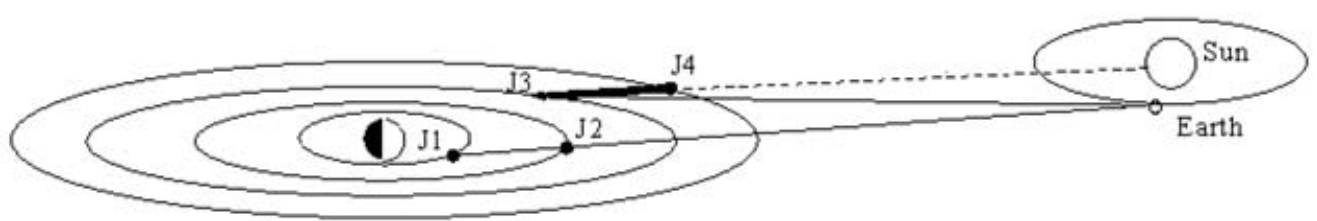

Figure 1. An eclipse and an occultation in the Jovian system.

The reduction of a mutual event observation provides a light curve; that means a time scale, and for each instant a photometric value, for which two quantities are important: the flux drop and the mid-light time. The flux drop is the maximum light-loss during the event, and the mid-light time is the instant corresponding to this light-loss. These two observed quantities may be linked to two geometrical ones: the distance minimum between the two satellites on the celestial sphere during the event, and the time corresponding to this distance (mid-time) which differs from the mid-light time by a few seconds due to light scattering by the surface of atmosphereless bodies, say all except Titan. 


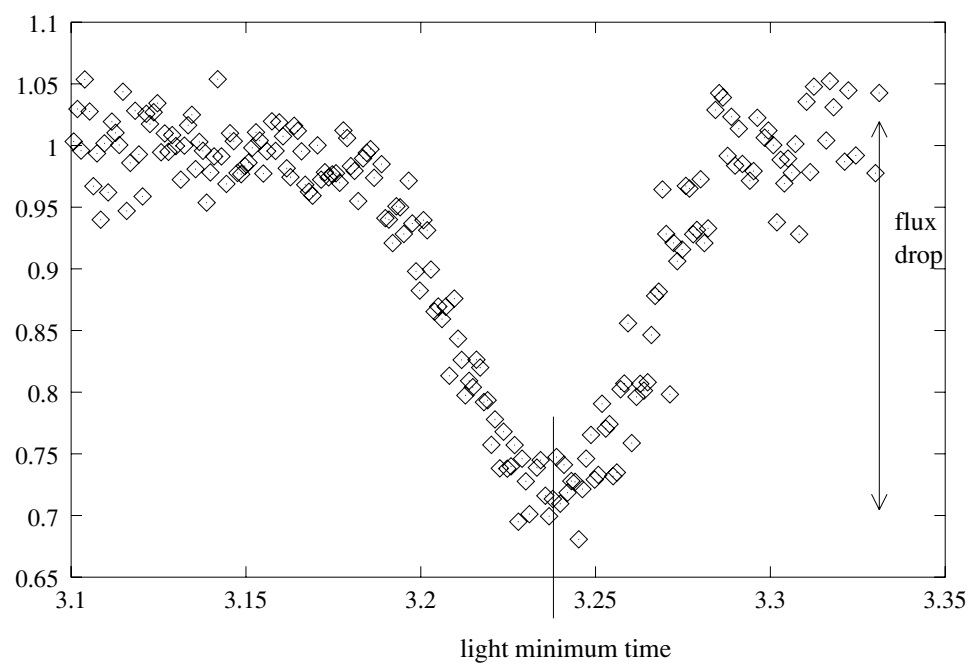

Figure 2. Observation of an occultation of S4 Dione by S3 Tethys at Pic-du-Midi Observatory on 21 September 1995. The flux is normalized and equal to 1 before and after the event. The abscissa is expressed in hours.

\section{Why observe mutual events?}

The mutual event observations have several advantages with respect to classical astrometric observations, for instance:

- high accuracy These observations are photometric instead of astrometric, so they are less dependant to the atmosphere turbulence.

- easy to observe Mutual events can be observed anywhere, even at low altitude sites, because some natural satellites, like the Galilean ones, are very easy to observe, their magnitudes being between 4 and 6 . Since the data are easy to register, amateurs can take part in observation campaigns.

- ephemerides improvement One of the goals for getting such accurate observations deals with ephemerides improvement. Indeed, besides spacecraft needs, it is necessary to predict star occultations by natural satellites (see, e.g., Sicardy et al. 1999), or to detect a secular accelerations in the motion of these bodies, consequences of tidal effects.

\section{Reduction method}

The method consists of modelling a theoretical light curve depending on some parameters, and to adjust them, for instance by a non-linear least squares fitting. The first main point is to model the diffusion of solar light by the surface of the satellites. There are several classical laws:

- Lambert law This law represents an isotropic diffusion of the light; it is reliable for Titan, which has an atmosphere. This law does not introduce any shift between mid-time and mid-light time.

$$
\frac{I}{F}=\frac{3}{2} p \cos i
$$


- Minnaert law This is an empirical law introducing a limb-darkening parameter $k$, see Minnaert (1961) for further details.

$$
\frac{I}{F}=B(\cos i)^{k(p)}(\cos e)^{k(p)-1}
$$

- Buratti-Veverka law This law takes into account reflection by secondary sources on the body's surface. It comes from Lommel-Seeliger law which has been extended in Buratti \& Veverka (1983).

$$
\frac{I}{F}=A \frac{\cos i}{\cos i+\cos e} f(p)+(1-A) \cos i
$$

In this equation, $f$ is a second-degree polynomial function. We used this law in Noyelles, Vienne \& Descamps (2003) for S-1 Mimas to S-5 Rhea with photometric parameters taken from Buratti \& Veverka (1984) and Devyatkin \& Miroshnichenko (2001).

- Hapke law This law is not described in detail here, due to its complexity and to the fact that we did not use it. This is a theoretical law which has two expressions depending on the roughness of the body's surface, see Hapke (1984) and Hapke (1986) for further details.

In all these formulae, $\pi F$ is the solar incident flux on a surface unit, $I$ the incident flux, $p$ the albedo, $i$ and $e$ respectively the incidence and emergence angles of the solar light on the body's surface (see Fig. 3), $k$ the limb darkening, and $A$ and $B$ photometric parameters depending on the body.

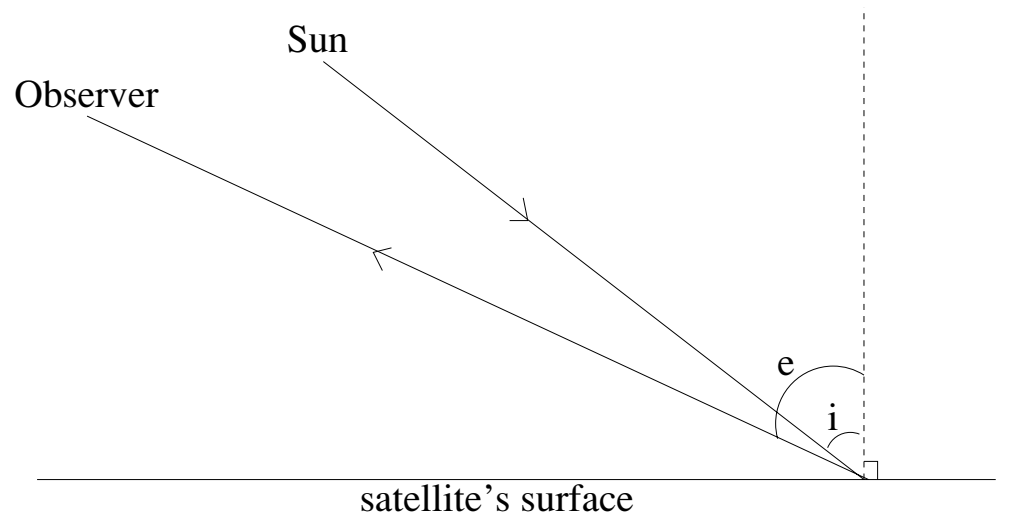

Figure 3. Description of the incidence angle $i$ and the emergence angle $e$.

In the case of an eclipse, there is a little difficulty for estimating the incident solar flux for a surface located in the penumbra zone, because the solar luminosity is not uniform on the solar disk, we have to take the solar limb darkening into account. For this purpose, we used an empirical formula given by Hestroffer \& Magnan (1998) giving a wavelength dependency of this darkening:

$$
I(r)=\mu^{\alpha}
$$




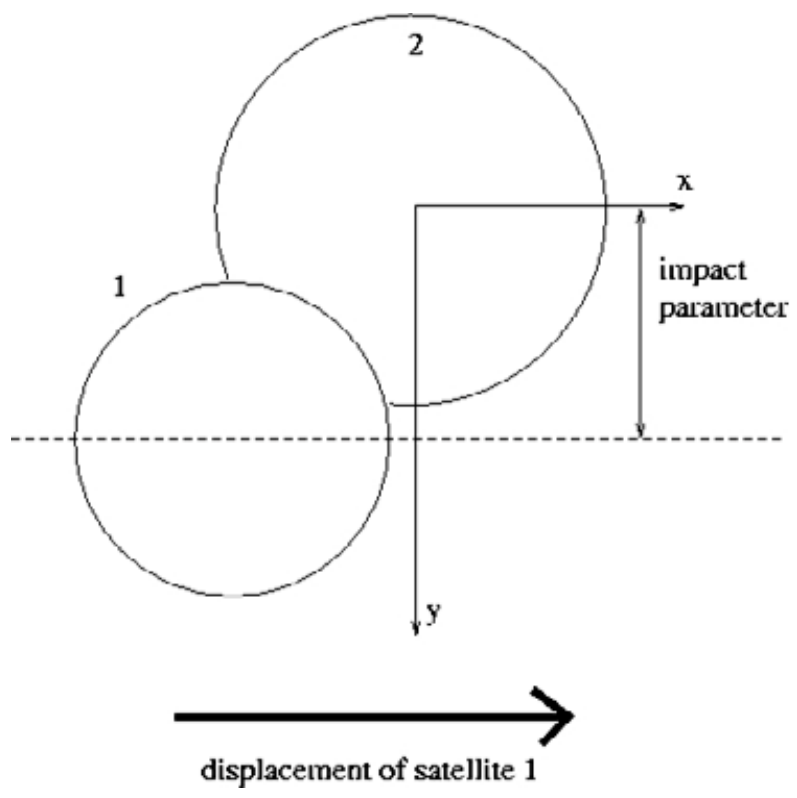

Figure 4. Geometrical representation of the impact parameter.

$$
\text { where: }\left\{\begin{array}{l}
\alpha \sim-0.023+0.292 \lambda^{-1} \text { if } \lambda \lesssim 2.4 \mu m^{-1} \\
\alpha \sim-0.507+0.441 \lambda^{-1} \text { if } \lambda \gtrsim 2.8 \mu m^{-1} \\
\lambda \text { wavelength in } \mu m \\
\mu=\sqrt{1-r^{2}} \\
r \quad \text { distance to the Sun's centre, } R_{\odot}=1
\end{array}\right.
$$

Once the detected flux is modelled, we have to model the motion too. We considered that, during the event, the further satellite did not move and the nearest moved on a straight line like a train on a railway with a constant speed (see Fig. 4), this model gives good results when there is no elongation.

After modelling the theoretical event, the last step is to adjust it to the observed light curve (see Fig. 5). The fit gives us a mid-time and an impact parameter, linkable to differential astrometric coordinates $(\Delta \alpha \cos \delta, \Delta \delta)$.

\section{Results}

\subsection{Case of the Saturnian satellites}

The first astrometric results of Saturnian satellites were published by Aksnes et al. (1984) after observing 14 mutual events in 1980 around the world, and reducing without taking account of the anisotropy of light scattering by the surface of the satellites. Fifteen years later, the PHESAT95 campaign organized by the actual IMCCE collected 65 light curves (see Thuillot et al. 2001). In Noyelles, Vienne \& Descamps (2003) we obtained 16 positions in which we are very confident and 32 in which we are confident, and re-reduced some light curves performed in 1980 in Japan by Soma \& Nakamura (1982) already reduced by Aksnes et al. (1984). The residuals for the best observations are about $20-30$ mas. 


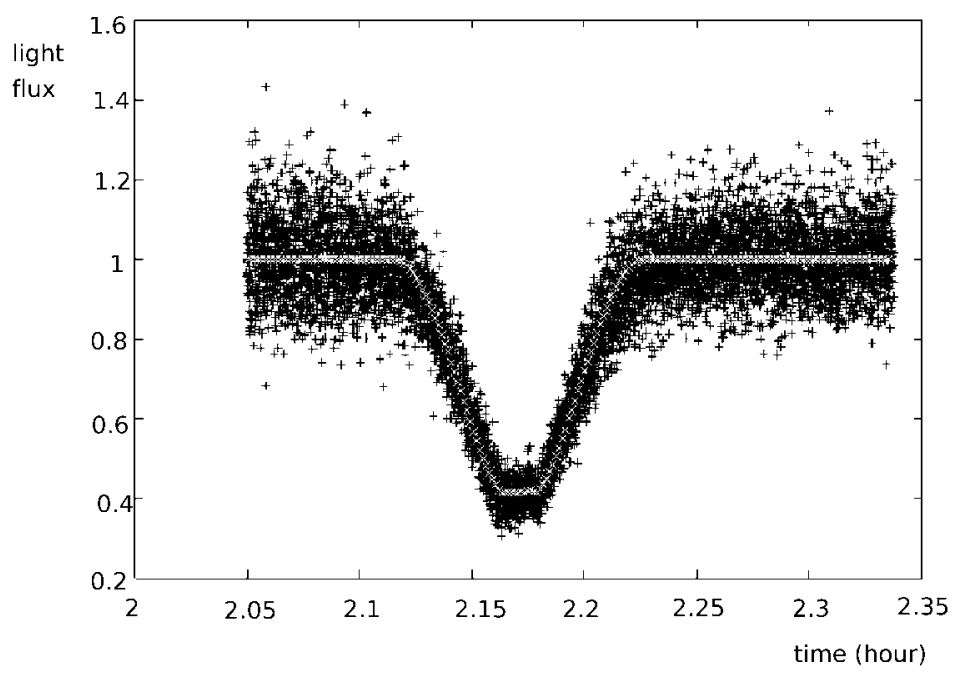

Figure 5. Fit of a theoretical light curve (bright curve) to an observed one (dark curve).

\subsection{Case of the Galilean satellites}

Now six observational campaigns have been done. Although the data are not reduced yet for the last one in 2002/2003, most observations have been introduced in the fit to the theories. For instance, Fig. 6 shows the residuals of PHEMU campaigns of 1985 and 1991 using L1 ephemerides (Lainey, Duriez \& Vienne 2004). One can see that the residuals are clearly below $0 . \prime 1$ (which is the mean of the $(\mathrm{O}-\mathrm{C}) \mathrm{s}$ for the astrometric observations).

\section{Improvements in the near future}

\subsection{Uncertainty in the position angle $( \pm \pi)$}

The impact parameter determination gives us only the separation angle between the two satellites at mid-time. The ephemerides give us good accuracy for the direction of the relative motion of the two satellites. But when the impact parameter is small, it can be difficult to determine the position angle $p$ between its actual value and $p \pm \pi$, more particularly for the Saturnian satellites, because the ephemerides are not as accurate as for the Galilean ones. In the future, the improvements in the dynamical theories will discriminate the good configuration.

\subsection{Knowledge of the satellites' photometry}

Thanks to the Galileo spacecraft mission, albedo maps of the four Galilean satellites are now available (see Vasundhara, Arlot, Lainey \& Thuillot 2003 for details, and Fig. 7 for an example). It is clear that the albedo variation of the satellite's surface modifies the light curve in a non negligible way (considering the already high precision involved). The introduction of such an effect has been recently done in the PHEMU97 campaign, and is still in progress for the other ones. The expected improvements of such correction is estimated around $0 .{ }^{\prime \prime} 02$. 


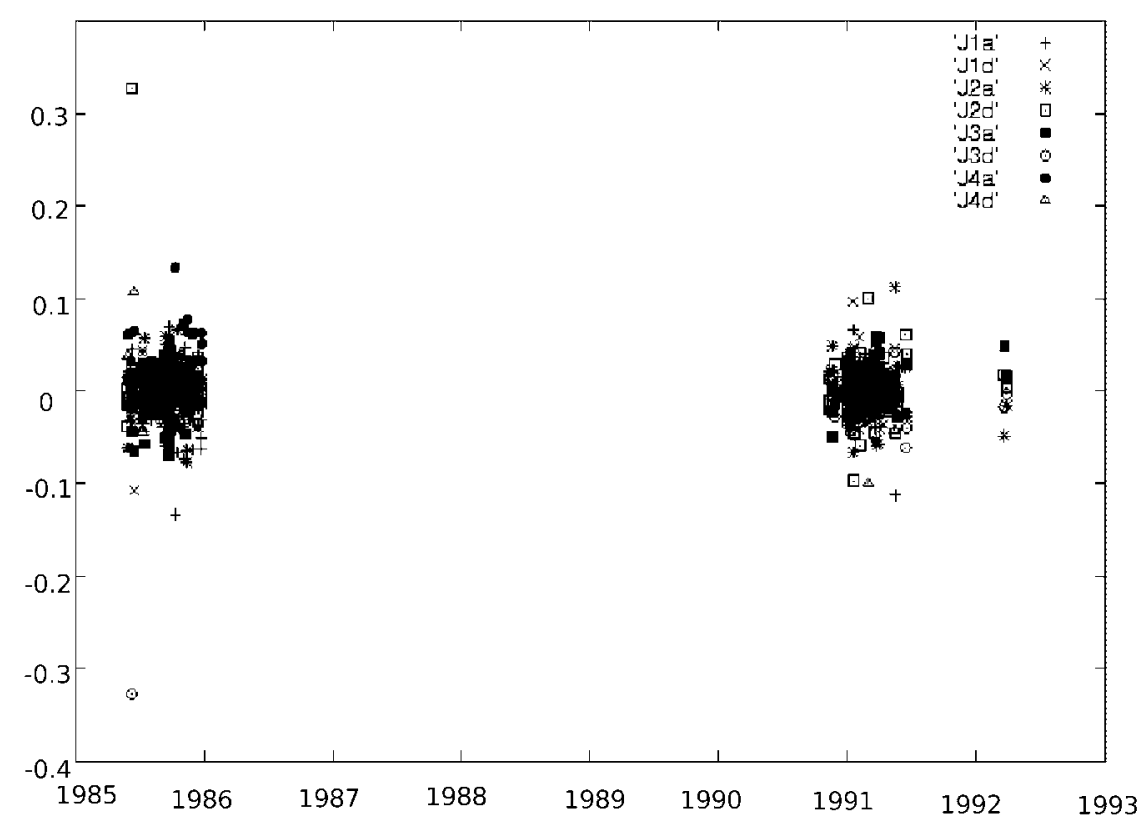

Figure 6. The (O-C)s of 1985 and 1991 PHEMU campaigns using L1 ephemerides. Y axis is expressed in arc seconds.

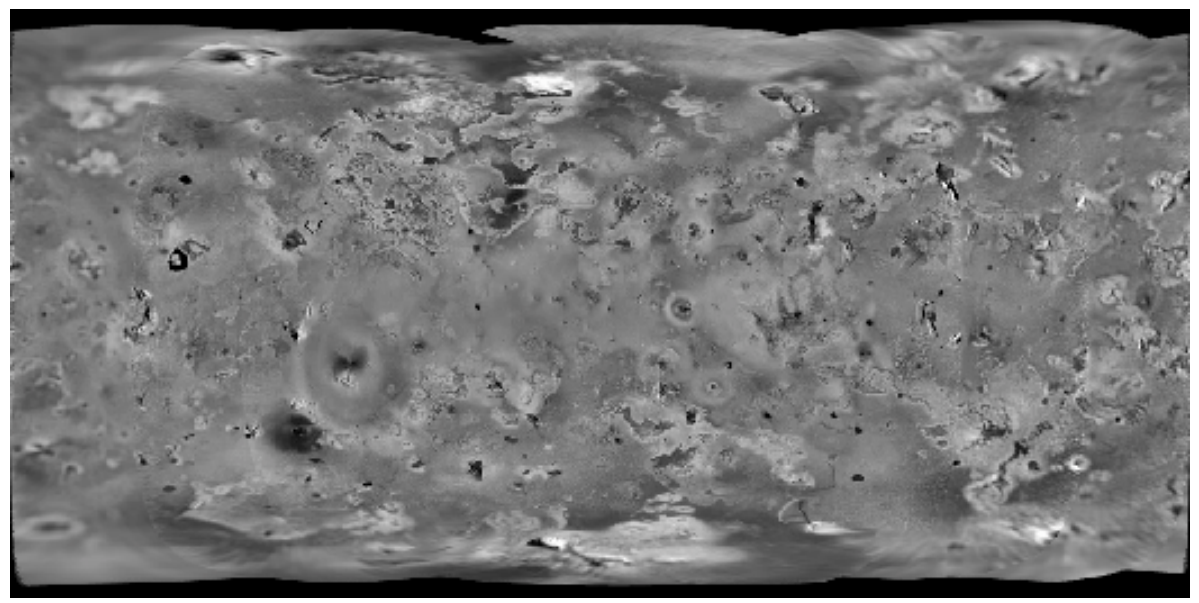

Figure 7. Io's albedo map (Geissler et al. 1999).

\subsection{Instrumental problems}

We said above that these observations are easy to perform; in fact it is easy to make good observations, but difficult to make very good ones, because some points are critical:

- The first one is the time reference. Lots of observers use their computer's clock, which has an accuracy not better than one second (inducing an error of around $10 \mathrm{~km}$ ). Better 
accuracy would help to detect a shift in longitude, that means a secular acceleration coming from tidal effects.

- The second one is the accuracy of the photometry. The exposure has to be short enough to not saturate the detector, and it is better to have a comparison object in the field, which has constant luminosity, to correct the variations in atmospheric transparency.

- The last one is the photometer calibration. Indeed, the light drop is strongly correlated with the detection threshold. It seems very difficult to correct this effect as it completely depends on the detector.

\section{Conclusion}

The main interest in observing mutual events is that no other observation is as accurate. They are useful to detect small shifts in ephemerides - that means secular acceleration in the mean longitudes, induced by tidal effects. Such detections would provide better knowledge of the internal structure of the satellites, for instance the depth of oceans, where life could have appeared. The determination of these effects is still in progress.

The next campaigns are in 2009 (PHEMU) and 2010 (PHESAT). We encourage observers over the whole world to take part of it and we also hope that for the first time, mutual events involving Uranian satellites will be observed in a few years.

\section{Acknowledgements}

The authors are indebted to R. Vasundhara (IIA, India), J.-E. Arlot, P. Descamps and W. Thuillot (IMCCE, Paris) and N. Emelianov (SAI, Russia) for fruitful discussions.

\section{References}

Aksnes, K., Franklin, F., Millis, R. et al. 1984 Astronomical Journal 89, 280-288.

Arlot, J.-E., Ruatti, C., Thuillot, W. et al. 1997 Astronomy \& Astrophysics Supplement Series 125, 399-405.

Buratti, B. \& Veverka, J. 1983 Icarus 53, 93-110.

Buratti, B. \& Veverka, J. 1984 Icarus 58, 254-264.

Devyatkin, A.V. \& Miroshnichenko A.S. 2001 Astronomy Letters 27, 193-197.

Geissler, P. E., McEwen, A. S., Keszthelyi, L., et al. 1999 Icarus 140, 265-282

Hapke B.W. 1984 Icarus 59, 41-59

Hapke B.W. 1986 Icarus 67, 264-280

Hestroffer, D. \& Magnan, C. 1998 Astronomy \& Astrophysics 333, 338-342.

Lainey, V., Duriez, L. \& Vienne, A. 2004 Astronomy \& Astrophysics 420, 1171-1183

Minnaert, M. 1961 in Planets and Satellites, ed. G.P.Kuiper \& B.M.Middlehurst (The University of Chicago Press) 213.

Noyelles B., Vienne A. \& Descamps P. 2003 Astronomy \& Astrophysics 401, 1159-1175

Sicardy, B., Ferri, F., Roques, F. et al. 1999 Icarus 142, 357-390.

Soma, M. \& Nakamura, T. 1982 Tokyo Astronomical Bulletin Second Series 267, 3039.

Thuillot, W., Arlot, J.-E., Ruatti, C. et al. 2001 Astronomy \& Astrophysics 371, 343-349.

Vasundhara, R., Arlot, J.-E., Lainey, V. \& Thuillot, W. 2003 Astronomy \& Astrophysics 410, $337-341$.

Vienne, A. \& Duriez, L. 1995 Astronomy \&3 Astrophysics 297, 588-605. 


\section{Discussion}

Mikhail Marov: Your talk is addressed to such effects in the solar system, but you mostly focused on the Galilean satellites. In recent years there has been an avalanche of discoveries of many new small-size satellites in the outer planets. Are there some applications of your model - your approach - in order to predict and observe such occultation effects for these new satellites?

Benoit Noyelles: Such satellites are faint, so observations are more difficult than, for instance, an occultation by a big satellite. For such faint ones the signal will be lost in the noise, so this observation would be difficult.

Mikhail Marov: I disagree. These satellites were discovered with ground-based facilities. It's a quite powerful technique right now. So if your theory can predict such events, it is possible to observe them.

Benoit Noyelles: Yes, but not so easy as the Galilean satellites.

Mikhail Marov: What kind of the model of scattering for the albedo features did you use? Lambert theory?

Benoit Noyelles: In fact, Lambert theory gives good results for Titan because it is has an atmosphere. But for the Saturnian satellites I use the Buratti-Veverka law which takes into account secondary reflections from the surfaces of the satellites. One can use the Minnaert law, but in this case it is better to fit the photometric parameters because it depends on the phase angle.

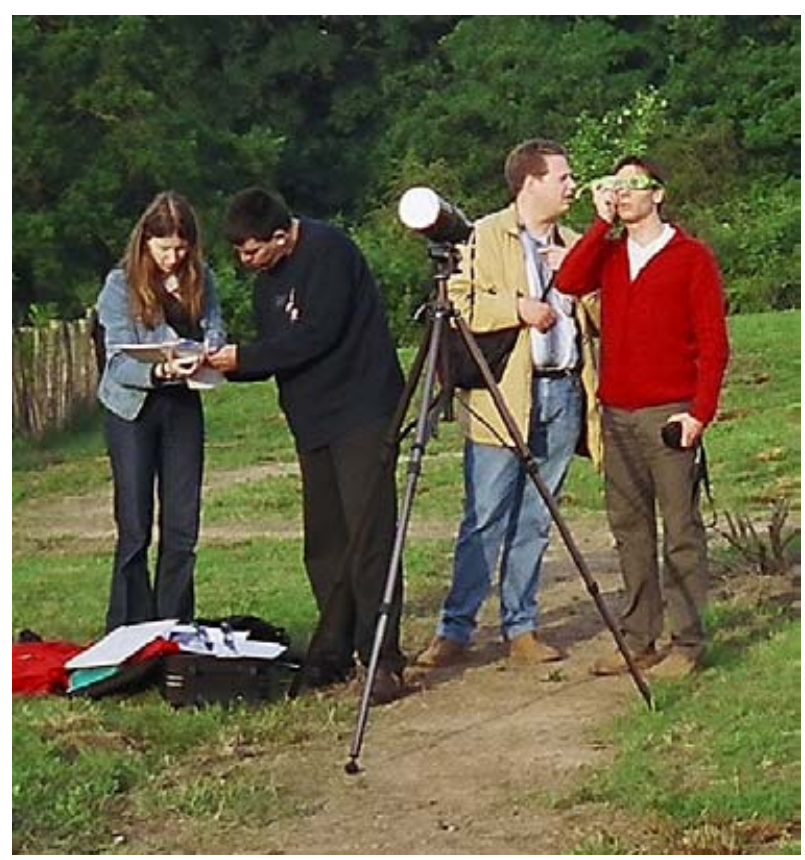

Sandrine d'Hoedt, Bernard De Saedeleer, Benoit Noyelles and Stephane Valk 